
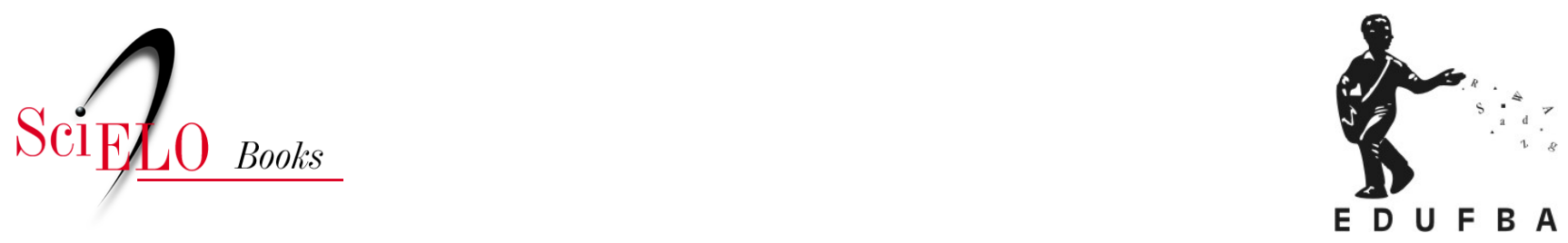

\title{
Policy \\ A utilização dos serviços odontológicos: elementos teóricos e conceituais
}

\author{
Denise Nogueira Cruz \\ Sônia Chaves \\ Maria Cristina Cangussu
}

CRUZ, D.N., CHAVES, S., and CANGUSSU, M.C. A utilização dos serviços odontológicos: elementos teóricos e conceituais. In: CHAVES, S.C.L. Política de saúde bucal no Brasil: teoria e prática [online]. Salvador: EDUFBA, 2016, pp. 203-225. ISBN 978-85-232-2029-7.

https://doi.org/10.7476/9788523220297.0008.

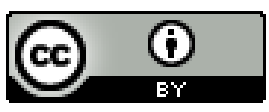

All the contents of this work, except where otherwise noted, is licensed under a Creative Commons Attribution 4.0 International license.

Todo o conteúdo deste trabalho, exceto quando houver ressalva, é publicado sob a licença Creative Commons Atribição 4.0. 


\title{
A utilização dos serviços odontológicos \\ ELEMENTOS TEÓRICOS E CONCEITUAIS ${ }^{1}$
}

\author{
Denise Nogueira Cruz \\ Sônia Chaves \\ Maria Cristina Cangussu
}

Os diferentes sistemas universais de saúde buscam, por meio da implantação de políticas e mecanismos de organização e provimento das ações e serviços, melhorar o nível de saúde das populações. No Brasil, a proposição de um sistema universal, integral e equânime foi inscrito na Constituição de 1988 conformando assim o Sistema Único de Saúde (SUS).

Pensar no debate sobre a melhoria da saúde das populações, inevitavelmente, faz emergir dois aspectos centrais: o indivíduo e sua relação com o meio em que vive e os serviços de saúde.

Quando se analisa a dimensão da relação entre o indivíduo e a sociedade, vários elementos podem ser levantados, desde aqueles que tratam de características genéticas, comportamentais, sociodemográficas e culturais até aos aspectos relacionados com a percepção sobre a necessidade de saúde e a busca e utilização dos serviços de saúde. Em relação aos serviços de saúde, é preciso identificar a existência (ou ausência) deles e o modo como estão organizados para responder as necessidades individuais e coletivas.

O presente capítulo foi desenvolvido a partir do texto que compõe a tese de doutorado Desigualdades na utilização dos serviços odontológicos: posição e tomadas de posição no espaço social, defendida por Cruz (2015). 
Neste capítulo, pretendemos introduzir uma reflexão teórico-conceitual sobre utilização dos serviços de saúde de modo a compreender a interface entre organização dos serviços e as necessidades de saúde; apresentar evidências das desigualdades no uso dos serviços odontológicos no Brasil e, finalmente, fomentar um debate sobre perspectivas metodológicas para o desenvolvimento de estudos acerca da utilização dos serviços de saúde.

\section{Elementos teóricos e conceituais}

Qualquer estudo sobre o uso dos serviços de saúde aponta para os conceitos de "necessidade", em suas variadas dimensões, "oferta" e "acesso", bem como o conceito de "utilização", propriamente dito. A "necessidade de saúde" se dá por meio de uma percepção individual e, nesse sentido, Schraiber e MendesGonçalves (1996) apontam que a definição de necessidades é algo complexo, podendo ser representada pela procura (como "demanda") por cuidados de saúde cuja origem é o "carecimento" dos indivíduos. Isto é, as pessoas, ao identificarem alguma alteração no seu estado de saúde, e que, para si, seja percebida como algo que carece e precisa de reestabelecimento, desencadeiam a procura pelos serviços configurando, assim, uma demanda.

Tais necessidades não aparecem sem história ou sem razão social e, desse modo, esses autores discutem o termo "social" para necessidades sociais em saúde. Afirmam que os "carecimentos" ou faltas são criados e recriados na vida em sociedade e, consequentemente, podem envolver muitas pessoas. Essa característica do termo social pode resultar em manipulação ideológica e política fazendo parecer que uma determinada necessidade que não é de todos seja mostrada como tal e, muitas vezes, assumida como prioridade. É preciso ainda chamar a atenção para o fato de que "essa situação de bem comum, ocorre ignorando a efetiva desigualdade das necessidades sociais". (SCHRAIBER; MENDES-GONÇALVES, 1996, p. 31)

Heller (1986), ao analisar a teoria das necessidades em Marx, define-a como "desejo consciente, aspiração, intenção dirigida a todo o momento para certo objeto e que motiva a ação como tal". (HELLER, 1986, p. 170) A partir do arcabouço teórico utilizado, define três grupos de necessidades: naturais ou físicas, sociais e radicais. Acrescenta que necessidades radicais não são necessidades de reprodução social, como as duas primeiras; são aquelas que efetivam o enriquecimento humano e associam-se aos processos de criatividade e liberdade. Para a autora, as necessidades de saúde relacionam-se com os dois primeiros 
grupos de necessidades, pois são fundamentais para manutenção da vida e, por serem socialmente produzidas, exigem a conformação de instituições (de educação, saúde e cultura, por exemplo) para satisfazê-las.

Portanto, as necessidades de saúde podem gerar uma demanda pelos serviços de saúde por parte de indivíduos ou grupos populacionais que pretendem melhorar sua condição de saúde, tratar uma doença ou solucionar determinado infortúnio. Elas também podem ser construídas socialmente e, também, manejadas política e ideologicamente, não correspondendo à compreensão do bem comum.

Quando analisamos a demanda pelos serviços de saúde, geralmente o debate volta-se para os aspectos relacionados aos próprios serviços, sua existência e organização para responder às necessidades de saúde da população e, necessariamente, volta-se aos conceitos de "acesso" e "utilização".

Para Travassos e Martins (2004), os conceitos de acesso e utilização são complexos e muitas vezes empregados de maneira imprecisa e variam de acordo com o autor. Adota-se o sentido conferido por Donabedian (1998) que emprega o substantivo "acessibilidade" para referir-se às características dos serviços que facilitam ou dificultam a sua utilização por parte dos usuários e, ainda, aponta duas dimensões da acessibilidade: barreira geográfica e barreira sócio-organizacional.

A barreira geográfica relaciona-se, por exemplo, com a distância dos serviços de saúde para que o indivíduo possa acessá-lo, a topografia do terreno com a presença de aclives e declives, escadarias além do custo e o tempo de locomoção. A barreira sócio-organizacional abrange aspectos sobre o modo como os serviços de saúde se organizam e ofertam os serviços, incluindo, por exemplo, mecanismos de triagem, organização da porta de entrada, fluxo, entre outros.

Entende-se que uma das maneiras de se medir o acesso aos serviços de saúde é a partir da sua "utilização", ato do uso do serviço, que, por sua vez, está relacionada aos fatores de ingresso nos serviços de saúde. Andersen (1995) denominou a etapa de utilização dos serviços de saúde como acesso realizado.

A utilização dos serviços de saúde objetivada na forma de consumo de serviços de saúde é mediada pelas necessidades de saúde e pelo comportamento dos indivíduos; bem como pelas características da oferta desses serviços. (SCHRAIBER; MENDES-GONÇALVES, 1996; TRAVASSOS; MARTINS, 2004) Há, portanto, um forte imbrincamento entre os conceitos de "acessibilidade", "oferta" e "utilização". 
A organização dos serviços de saúde, na tentativa de responder às necessidades de saúde da população, também pode ser analisada em função do caráter das políticas de saúde e da extensão/tipos de cobertura dos sistemas de saúde (seguro saúde, cobertura universal, entre outros). Nesse sentido, é importante compreender que o Estado responde às necessidades de saúde da população e implementa diferentemente suas políticas de saúde compreendidas, aqui, como "ação ou omissão do Estado, enquanto resposta social, diante dos problemas de saúde e seus determinantes, bem como da produção, distribuição e regulação de bens, serviços e ambientes que afetam a saúde dos indivíduos e da coletividade humana". (PAIM, 2003, p. 588)

Assim, ao introduzir o componente da política nesse debate e estabelecer as necessidades em saúde como uma construção social, pode-se retomar algumas questões apresentadas. Tratou-se, anteriormente, que as necessidades sociais podem ser construídas como necessidades pelas camadas privilegiadas ou dominantes. Portanto, os mecanismos, as técnicas e processos para criação das instituições que satisfaçam necessidades sociais não são neutros. Desse modo, cabe questionar quais necessidades são priorizadas quando uma determinada política, um programa ou até mesmo um serviço de saúde é implantado e se ele atende, de fato, às "reais" necessidades de saúde de uma população.

Compreender o processo de implementação das políticas de saúde e quais necessidades serão respondidas pode revelar aspectos que confirmam a compensação ou o reforço das desigualdades de acesso e utilização dos serviços de saúde. Percebe-se, portanto, que a aplicação dos conceitos aqui apresentados possibilita a construção de estudos no âmbito da saúde com perspectivas de análise distintas.

\section{Evidências sobre a utilização dos serviços odontológicos e a Política Nacional de Saúde Bucal (2004)}

A utilização dos serviços odontológicos tem sido objeto de investigações nacionais e internacionais. Metodologicamente, destaca-se o predomínio da abordagem epidemiológica e a utilização de bases de dados provenientes de inquéritos populacionais nacionais (BARROS; BERTOLDI, 2002; MANHÃES; COSTA, 2008; MIRANDA; PERES, 2013; PERES et al., 2012a; SILVA et al., 2011) e internacionais (BAKER, 2009; GUARNIZO-HERREÑO et al., 2003; MATSUYAMA et al., 2014; PALÈNCIA et al., 2014). Há também estudos que utilizaram dados prove- 
nientes de coortes prospectivas (ASTROM et al., 2013; TCHICAYA, LORENTZ, 2014) e, inclusive, através do inquérito telefônico. (PERES et al., 2012b)

Os estudos sobre a utilização de serviços odontológicos no Brasil apontam desigualdades no padrão de utilização com associações, principalmente, entre: a) nível econômico, com maior acesso para aqueles de maior renda (ARAÚJO et al., 2009; BARROS; BERTOLDI, 2002; CAMARGO; DUMITH; BARROS, 2009; DAVOGLIO et al., 2009; MANHÃES; COSTA, 2008; PERES et al., 2012a); b) faixa etária, especialmente os adultos jovens e mulheres (ASTROM et al., 2013; BARROS; BERTOLDI, 2002; PERES et al., 2012a); e c) nível de escolaridade, com maior acesso aos mais escolarizados. (ARAÚJO et al., 2009; CAMARGO; DUMITH; BARROS, 2009; MANHÃES; COSTA, 2008; MIRANDA; PERES, 2013)

No Quadro 1, são apresentados os principais estudos nacionais publicados entre 2009-2015 e indexados com os descritores "utilização; assistência odontológica" e a síntese dos seus resultados mais relevantes (Quadro 1).

$\mathrm{Na}$ investigação de Araújo e colaboradores (2009), a maior utilização dos serviços odontológicos estava associada com o maior nível econômico e de escolaridade e com a melhor autopercepção de saúde. Outro estudo de base populacional, utilizando dados da Pesquisa Nacional por Amostra de Domicílios (PNAD), também verificou uma maior proporção de indivíduos que nunca consultou o dentista no grupo de indivíduos que representava o quintil mais pobre, sendo que, no grupo de indivíduos de 20-49 anos, a proporção de pessoas desse quintil que nunca foi ao dentista foi 16 vezes maior do que no quintil mais rico. (BARROS, BERTOLDI, 2002)

Estudo de Manhães e Costa (2008), que analisou o uso de serviços odontológicos em três grupos etários (15-19 anos, 35-44 anos e 65-74 anos), apontou que indivíduos menos escolarizados (jovens e adultos) e com menor renda per capita (jovens e idosos) têm limitações de acesso. Entre os adultos (35-44 anos), as chances dos homens utilizarem o serviço foi quase metade daquela observada entre as mulheres. Alguns fatores podem estar associados a esses resultados, como, por exemplo, a inadequação da oferta em relação à disponibilidade de horário do adulto produtivo brasileiro.

No Brasil, a PNAD, em 2003, verificou que $18,7 \%$ dos brasileiros nunca haviam consultado um dentista e apontou desigualdades na utilização dos serviços odontológicos, já que a proporção de pessoas que nunca consultaram um dentista era maior entre os brasileiros mais pobres. (IBGE, 2005) 
Entende-se que estudos sobre a utilização de serviços odontológicos e as desigualdades nessa utilização precisam também ser relacionados com o quadro epidemiológico da condição de saúde bucal da população brasileira. Dados epidemiológicos evidenciam a cárie dentária como o problema de saúde bucal mais prevalente no país. (BRASIL, 2004a) Esse agravo, associado à falta de acesso aos serviços de saúde bucal, resulta em uma alta prevalência de perda dental e grande necessidade de tratamentos especializados em adultos jovens e idosos. (FIGUEIREDO; GOES, 2009; FRAZÃO; NARVAI, 2010)

Os inquéritos epidemiológicos nacionais apontam um considerável aumento na média de dentes permanentes acometidos pela cárie com o aumento da idade; particularmente nas faixas etárias de 15 a 19 anos e 35 a 44 anos, resultando em um CPO-D2 de 2,8 aos 12 anos de idade e de 6,2 e 20,1 nos outros dois grupos etários respectivamente. (BRASIL, 2004b) Destaca-se, ainda, que o percentual do componente perdido no grupo de 35 a 44 anos corresponde a mais de $65 \%$ do CPO-D, o que é fortemente agravado na faixa etária de 65-74 anos. O levantamento realizado em 2010 (BRASIL, 2011) indica uma melhora na condição da saúde bucal de modo que o CPO-D nos grupos etários investigados alcançou a média de 2,07 nas crianças de 12 anos. Entretanto, permanece o aumento na média de dentes permanentes acometidos pela cárie com o aumento da idade resultando em um percentual de $68,8 \%$ dos indivíduos com idade entre 35 a 44 anos necessitando de alguma prótese dentária. (BRASIL, 2011)

A Política Nacional de Saúde Bucal (PNSB), lançada em 2004, apresenta estratégias para o enfrentamento dessas necessidades de saúde bucal da população brasileira. Essa política tem como principais propostas reorganizar os serviços públicos odontológicos com reorientação do modelo de atenção, qualificar e fortalecer a Estratégia Saúde Família (ESF) e ampliar o acesso aos procedimentos especializados, também, denominada atenção especializada. (BRASIL, 2004a)

A PNSB é considerada um importante avanço, visto que é uma política que procurou romper com as ações focalizadas pretendendo uma integralidade da atenção. No Brasil, o modelo de atenção à saúde bucal foi, historicamente, com ênfase curativo-restaurador e mutilador e elegeu, como público-alvo prioritário, a população escolar. A primeira oferta sistemática de serviços foi ao interior

2 O CPO-D é um índice que expressa a média de dentes permanentes cariados, perdidos e/ou obturados em virtude da doença cárie. É um indicador utilizado para avaliar o ataque da doença cárie numa determinada população. 
do Sistema Incremental de Atendimento, introduzido em 1952 pela Fundação SESP (Serviço Especial de Saúde Pública), em Aimorés, Minas Gerais, baseado na assistência odontológica para escolares de forma programada e sistemática, com o objetivo de aumentar ao máximo a produtividade, na forma de número de pacientes atendidos, e reduzir as necessidades de tratamento em crianças de 6 a 14 anos. Esse modelo de oferta programática também pressupunha a complementariedade de uma fase preventiva, com aplicações tópicas de flúor até o início da década de 1970 e, posteriormente, o uso de bochechos fluorados, além da recomendação da fluoretação das águas de abastecimento público. (DINIZ, 1987; MARTILDES, 1992) Para a população adulta, restava a demanda espontânea clássica, centrada basicamente na mutilação.

A possibilidade de uma estratégia racionalizadora na prestação da assistência odontológica fez com que o sistema incremental se transformasse num modelo de atendimento no Brasil, até a década de 1980, focalizando prioritariamente crianças de 7 a 12 anos de idade, especialmente nos programas de odontologia escolar. Entretanto, avaliações realizadas sobre o sistema, em municípios dos estados da Bahia e São Paulo, não identificaram impacto epidemiológico na redução dos índices de cárie dentária em relação às crianças que não possuíam nenhuma assistência odontológica sistemática, como relataram os estudos de Diniz (1987) e Martildes (1992).

A maior crítica ao sistema incremental foi de uma forma geral, a falta de eficácia da fase preventiva do programa, que não incorporou de forma significativa a educação sanitária e a evolução do conhecimento científico e técnico da odontologia preventiva, perpetuando uma assistência individual e curativa, de baixo impacto epidemiológico. (DINIZ, 1987; MARTILDES, 1992; NARVAI, 1994) Configurou, ainda, uma exclusão da maior parte da população, que dependia de políticas que privilegiavam a prática clínica privada, de alta tecnologia, cara e inacessível à população, já que, no setor público, predominava a assistência mutiladora e emergencial, restrita à remoção de focos infecciosos.

Na década de 1980, relatava-se o grave perfil epidemiológico da população brasileira, resultado de um modelo de atenção ineficaz e ineficiente, onde predominava a descoordenação, má distribuição da rede assistencial e dos recursos, baixa cobertura, enfoque curativo e o caráter mercantilista e monopolista do atendimento odontológico, aspectos descritos na I Conferência Nacional de Saúde Bucal (BRASIL, 1986), mesmo com o registro de algumas experiências 
municipais positivas, com ênfase nas necessidades de saúde da população e descentralização dos serviços.

O debate sobre os modelos de atenção e a consolidação de um novo referencial teórico-metodológico na determinação do processo saúde-doença bucal ganha amplitude com o movimento da Reforma Sanitária Brasileira, na busca por um modelo de prática que incluísse a saúde bucal no campo das demandas e necessidades de saúde da população e que tivesse resposta e respaldo no setor público. Assim, e somente após mais de uma década da conformação do SUS, a inserção da odontologia no então Programa de Saúde da Família (PSF) surge como uma iniciativa promissora para a inversão do modelo de atenção e como resposta às necessidades em saúde bucal no âmbito da atenção primária. A partir da PNSB de 2004, é que, além da atenção básica, priorizando a Equipes de Saúde da Família. (ESF) como forma de organização das práticas, também assume-se como política de saúde a necessidade de reabilitação do adulto e a sua inclusão em todos os níveis de atenção. (BRASIL, 2004b)

No que diz respeito às evidências sobre o efeito da ESF na utilização dos serviços odontológicos, identificam-se poucos estudos nacionais. Avaliações sobre a utilização de serviços odontológicos em áreas cobertas pela ESF foram realizadas em alguns municípios brasileiros. (BALDANI et al., 2010; BALDANI; ANTUNES, 2011; OLIVEIRA et al., 2014; PEREIRA et al., 2009; ROCHA; GOES, 2008) Em Ponta Grossa, no Paraná, a investigação sobre a existência de desigualdades no acesso e utilização de serviços odontológicos, para indivíduos residentes em áreas cobertas pela ESF, identificou que 15\% nunca tinham realizado consulta odontológica e esse percentual chegou a 50\% entre as crianças de o-6 anos. (BALDANI, ANTUNES, 2011) Nesse estudo, o fato de ser coberto por ESF não foi associado às recentes consultas ao dentista e melhor condição socioeconômica esteve positivamente associada à visita recente ao dentista. $\mathrm{Ou}$ seja, a implementação das Equipes de Saúde Bucal (ESB) na ESF não significou, enquanto política de saúde, maior utilização e as desigualdades persistiram.

Rocha e Goes (2008), ao compararem o acesso de serviços de saúde bucal em áreas cobertas e não cobertas pela ESF, identificaram que não há associação entre residir em uma ou outra área e que o percentual de indivíduos que procurou o dentista do serviço público $(34,5 \%)$ foi muito inferior ao daqueles que procuram serviços privados ou conveniados (64,3\%). Esses autores também identificaram que, entre aqueles que utilizaram o serviço público, apenas $6,8 \%$ tiveram acesso ao dentista da ESF. O estudo de Olinda, Pernambuco, obser- 
vou, da mesma forma, uma baixa utilização $(18,9 \%)$ dos serviços odontológicos prestados pela unidade de saúde da família por parte da população adscrita. (OLIVEIRA et al., 2014)

O ensaio comunitário realizado em Natal, Rio Grande do Norte, revelou que o impacto da saúde bucal no PSF na utilização de serviços odontológicos só é significativo quando se toma por análise áreas sem nenhuma cobertura ou quando cobertas por unidades tradicionais sem PSF ou Programa de Agentes Comunitários de Saúde. (PEREIRA et al., 2009) No Maranhão, foi observada uma baixa utilização dos serviços de saúde bucal por crianças e adultos incoerente com a cobertura da ESF no estado, que era de 74,29\% (GOMES et al., 2014), persistindo às iniquidades em saúde na população. No âmbito da atenção primária em odontologia, portanto, percebe-se que a política de uma crescente implantação de ESB, por si só, não tem sido capaz de garantir maior utilização dos serviços.

No que diz respeito à atenção especializada, a PNSB buscou viabilizar a oferta de procedimentos especializados através dos Centros de Especialidades Odontológicas (CEO) e Laboratórios Regionais de Próteses Dentárias. (BRASIL, 2004a) Os CEO são estabelecimentos de saúde que devem ofertar os serviços de diagnóstico bucal, com ênfase no diagnóstico e detecção do câncer de boca; periodontia especializada; cirurgia oral menor dos tecidos moles e duros; endodontia e atendimento a portadores de necessidades especiais. (BRASIL, 2004a)

Há de se conceber, entretanto, que, para garantir a integralidade, é necessário repensar a atenção primária e o estabelecimento de adequadas estratégias de referência e contrareferência, ainda frágeis. Do ponto de vista da sua cobertura, utilização e efetividade, também, foram encontrados poucos estudos com foco na atenção especializada em odontologia no Brasil.

Investigações que avaliaram a implementação da PNSB no âmbito da atenção especializada apontam que os resultados da utilização dos serviços desse nível de atenção não se distanciam daqueles que investigaram áreas cobertas pela ESF, visto que a atenção especializada depende, necessariamente, do funcionamento adequado da atenção primária.

O estudo de Chaves e colaboradores (2010), realizado na Bahia, identificou que os CEO realizavam atendimento à livre demanda e, predominantemente, procedimentos básicos. Portanto, não garantiam a integralidade da atenção, devendo ser implantados em município com uma atenção primária adequada- 
mente estruturada. Nesse mesmo estudo, os resultados apontaram que usuários com facilidade de acesso geográfico, moradores de municípios com elevada cobertura do PSF, menores de 35 anos de idade e com necessidade de serviço endodôntico tiveram mais chances de completar tratamento especializado.

Outros estudos, acerca da avaliação dos CEO, desenvolvidos numa perspectiva nacional (GOES et al., 2012), estadual (FIGUEIREDO; GOES, 2009) e municipal (CHAVES et al., 2011; CHAVES et al., 2012; SOUZA; CHAVES, 2010), apontam um desempenho ruim de alguns desses estabelecimentos bem como baixa ou inadequada utilização dos serviços.

As contribuições apresentadas acerca da utilização dos serviços odontológicos demonstram persistências das desigualdades na utilização desses serviços e apontam lacunas quanto aos aspectos que expliquem a utilização dos serviços no que diz respeito aos determinantes sociais da saúde. ${ }^{3}$

No Brasil, apesar da pretendida cobertura universal dos serviços odontológicos, há questões que merecem aprofundamento investigativo, como as diferenças na utilização dos serviços odontológicos em relação aos demais serviços de saúde. Um inquérito realizado por Barros e Bertoldi (2002) identificou que $52 \%$ dos atendimentos não odontológicos são realizados no SUS, enquanto apenas $24 \%$ dos atendimentos odontológicos são SUS. Nesse estudo, a maior parte dos atendimentos odontológicos (69\%) ocorreu em serviços particulares e de saúde suplementar.

Além disso, Silva e colaboradores (2011), ao investigarem a utilização de serviços de saúde no SUS, nos anos de 2003 e 2008, identificaram que, diferentemente das consultas médicas, os serviços odontológicos no SUS correspondem a apenas um terço das consultas realizadas. Ou seja, mesmo com cobertura universal e ampliação dos serviços odontológicos no SUS, os serviços privados e conveniados correspondem pela maior parte da assistência odontológica aos brasileiros, e observa-se, principalmente nos grandes centros urbanos, crescimento significativo da saúde suplementar.

3 Determinantes Sociais da Saúde (DSS) correspondem às "condições em que as pessoas nascem, crescem, vivem, trabalham e envelhecem, incluindo aquelas relacionadas ao sistema de saúde. Essas circunstâncias são influenciadas pela distribuição de dinheiro, poder e recursos nos níveis global, nacional e local, que, por sua vez, é moldada pelas políticas vigentes. As iniquidades em saúde são causadas, em sua maioria, pelos DSS. O termo é utilizado para resumir as forças sociais, políticas, econômicas, ambientais e culturais mais amplas que influenciam as condições de vida das pessoas". (OMS, 2011) 
Por fim, o estudo de Soares e colaboradores (2013) acrescenta mais um elemento a ser investigado: as desigualdades quanto ao tipo de serviço utilizado. Esses autores, ao investigarem as desigualdades na utilização dos serviços odontológicos, e assumindo a exodontia como tratamento mais resolutivo para população de baixa renda, observaram que a utilização desse procedimento foi associada a ser beneficiário de programa social, ter idade maior que 35 anos e menor nível de escolaridade. O estudo evidenciou que, mesmo entre grupos sociais relativamente homogêneos, há maior realização da exodontia entre os menos escolarizados e socialmente mais vulneráveis.

\section{Modelo explicativo de Ronald Andersen e perspectivas}

Para compreender a utilização dos serviços de saúde, modelos teóricos explicativos foram elaborados, a exemplo dos modelos de Andersen de 1968; Dutton de 1986 e Evans e Stoddart de 1990 (PAVÃO; COELI, 2008). Contudo, destaca-se que, na expressiva maioria dos estudos, nacionais e internacionais, que investigou a utilização de serviços odontológicos, predomina a aplicação do modelo elaborado por Andersen. (ADAY; ANDERSEN, 1974; ANDERSEN, NEWMAN, 1973; ANDERSEN, 1995)

No final da década de 1960, Andersen desenvolveu um modelo para compreender a utilização dos serviços de saúde. O autor revisitou o modelo inicial desenvolvendo mais outras três representações chegando àquele que denominou de fase emergente apresentado na Figura 1 adiante.

Inicialmente, o modelo comportamental era centrado na família e depois foi deslocado para o indivíduo. Na fase inicial, o desfecho era a utilização dos serviços (ANDERSEN, 1995) e o modelo sugeria que o uso dos serviços ocorria em função de determinantes individuais representados pelos seguintes fatores: propensão do indivíduo a utilizar os serviços (características ou fatores predisponentes), impedimentos ou facilidades para a utilização (fatores capacitantes) e as necessidades de saúde. (ANDERSEN; NEWMAN, 1973; ANDERSEN, 1995)

Os fatores predisponentes, no modelo da década de 1960 (ANDERSEN, 1995), estavam organizados em três grupos: características demográficas (idade, gênero, situação conjugal), estrutura/posição social (escolaridade, raça, religião, ocupação, tamanho da família) e crenças em relação aos serviços e cuidados de saúde (atitudes, valores e conhecimentos que afetam a percepção da necessidade e utilização). 
Os fatores capacitantes referiam-se aos meios disponíveis para utilizar o serviço, ou seja, àqueles fatores que irão capacitar ou facilitar a utilização dos serviços de saúde. São condições relacionadas ao indivíduo/família (como renda, cobertura de seguro) e à comunidade em que se vive (a natureza da fonte regular de cuidados de saúde, localização dessa fonte regular/rural-urbano, região do país, tempo de espera pelos cuidados de saúde).

As necessidades de saúde dizem respeito ao que ocasiona o imediato uso dos serviços de saúde. Podem ser necessidades percebidas pelo indivíduo (sintomas, estado geral) ou diagnosticadas, mediante avaliação profissional.

Na fase 2, do modelo o desfecho é a satisfação do usuário e não mais a utilização em si mesma. Nesse modelo, o sistema de saúde (com três dimensões: políticas em saúde, recursos financeiros, organização dos seviços) é incorporado como um outro determinante da utilização dos serviços. (ADAY; ANDERSEN, 1974; ANDERSEN, 1995) Portanto, nessa segunda representação, as características individuais (desenvolvidas na fase 1) e o sistema de saúde determinam o uso dos serviços que, por sua vez, podem resultar na satisfação do usuário.

$\mathrm{Na}$ fase 3, Andersen (1995), novamente repensa o desfecho do modelo que passa a ser mensurado pela satisfação do consumo e, também, pelo "acesso efetivo" (quando a utilização resulta em melhoria no estado de saúde ou satisfação com os serviços) e "acesso eficiente" (quando a melhoria no estado de saúde ou a satisfação com os serviços aumenta em relação ao volume de serviços utilizados). São introduzidos nessa terceira fase, o ambiente e as práticas individuais com a saúde. (ANDERSEN, 1995)

O modelo na fase 4 ou emergente (Figura 1) é aprimorado na tentativa de traduzir a complexidade de representar a utilização dos serviços de saúde. Nesse modelo, o ambiente é conformado pelo sistema de saúde e ambiente externo e são incorporadas algumas setas para demonstrar o quanto cada componente do modelo pode influenciar os demais (relações de feedback). 
Figura 1 - Modelo de utilização dos serviços de saúde elaborado por Andersen (fase 4 ou modelo emergente)

Ambiente Características da população Comportamento de saúde Desfechos

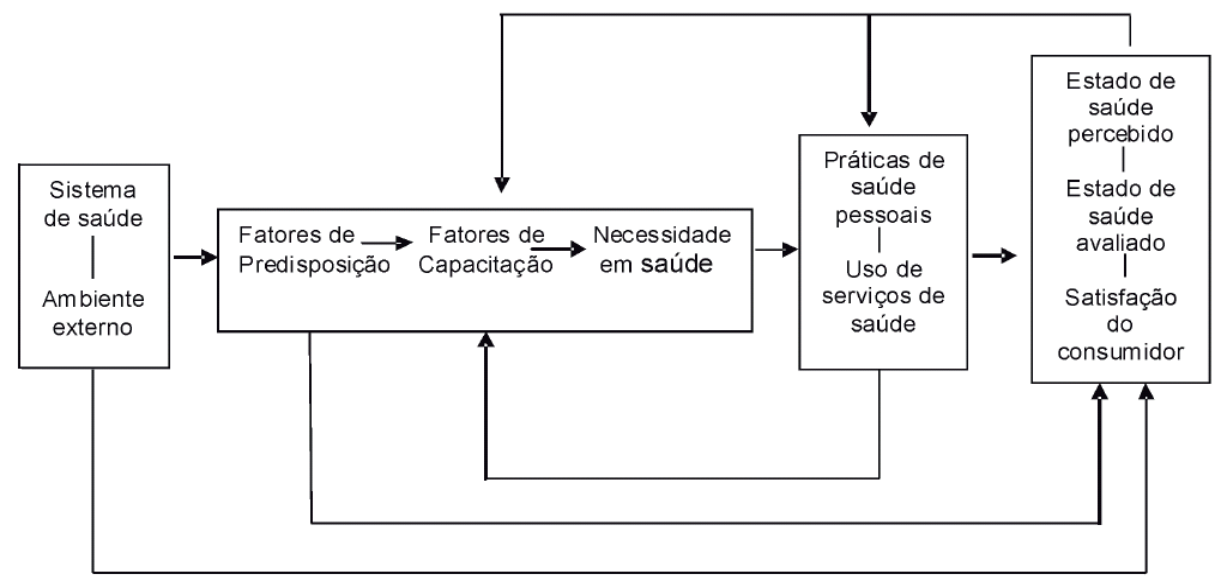

Fonte: Andersen (1995, p. 7, tradução nossa).

Entende-se que a utilização dos serviços de saúde pode ser explicada, em parte, pelo modelo proposto por Andersen, pela interação entre os determinantes individuais, do sistema de saúde e contexto social, além da experiência passada de utilização dos serviços, como apresentado em alguns estudos. (ASTROM et al., 2013; BAKER, 2009; BALDANI; ANTUNES, 2011; CAMARGO; DUMITH; BARROS, 2009; GOMES et al., 2014; MARSHMAN et al., 2012; MUIRHEAD, 2009; PINTO et al., 2012; ROCHA; GOES, 2008)

Essas investigações apontam importantes evidências sobre os fatores associados à utilização, entretanto, explicam parcialmente as razões das desigualdades na utilização dos serviços odontológicos. Nesse sentido, cabe ainda destacar que os estudos sobre utilização dos serviços odontológicos adotam, majoritariamente, uma abordagem epidemiológica, especialmente quando aplicam o modelo explicativo de Andersen. Outro elemento importante a ressaltar é a perspectiva teórico-marxista que fundamenta a explicação dos conceitos apresentados no início do texto por Heller (1986) e Schraiber e Mendes-Gonçalves (1996).

Propõe-se uma reflexão sobre como outras correntes sociológicas podem contribuir para futuras investigações sobre as desigualdades na utilização dos 
serviços de saúde a exemplo dos serviços odontológicos. Na direção desse debate, a teoria da ação social de Pierre Bourdieu pode revelar outras dimensões nos estudos do campo da saúde (VIEIRA-DA-SILVA, 2010) e, particularmente, sobre a utilização dos serviços de saúde e a relação entre a atenção à saúde bucal e a posição no espaço social dos agentes (CHAVES, VIEIRA-DA-SILVA, 2008; CRUZ, 2015), usuários desses serviços.

Os conceitos dos diferentes capitais (como o econômico, cultural e social), de habitus e campo (BOURDIEU, 2008, 2010) ajudam a explicar como a escolha pelo uso de determinados serviços de saúde podem ser a expressão de "disposições” social, histórica e inconscientemente incorporadas. Para Bourdieu, a posição ocupada pelo indivíduo no espaço social guarda relação com o modo com que o mundo é inscrito em seu corpo, ou seja, a maneira de andar, carregar o corpo, inclusive, a maneira de comer, revela um habitus, ou mais precisamente, uma hexis corporal. (BOURDIEU, 2007)

Estudos com tal abordagem teórica podem revelar como o uso dos serviços de saúde expressa, especialmente para as classes populares, um "gosto de necessidade" (necessidade econômica impõe a escolha pelo necessário) e como determinadas escolhas são a transformação de "necessidade em virtude". (BOURDIEU, 2008)

No estudo de Cruz (2015), o "gosto" pelo uso de determinados serviços odontológicos revelou-se diferente entre os indivíduos de estratos sociais mais elevados e aqueles das classes populares. O julgamento, por exemplo, do uso de dentes artificiais (prótese dentária) ao invés dos dentes naturais, como belo revelou-se como um gosto de classe que é reconhecido entre agentes com disposições e posições próximas no espaço social. (CRUZ, 2015) Esse estudo apontou, ainda, que a utilização de serviços odontológicos privados é uma prática distintiva e o encontro dos diferentes habitus (habitus do profissional e habitus do usuário) é importante para compreender as desigualdades no uso desses serviços.

Portanto, elementos da teoria social de Bourdieu apontam novas perspectivas de análise e revelam-se profícuas para outras explicações da persistência das desigualdades na utilização dos serviços odontológicos mesmo em condições de ampliação da rede de serviços públicos nessa área. 


\section{Referências}

ADAY, L. A.; ANDERSEN, R. A framework for the study of access to medical care. Health Services Research, v. 9, n. 3, p. 208-220, 1974.

ANDERSEN, R. Revisiting the behavioral model and access to medical care: does it matter?Journal of health \& social behavior, v. 36, March, p.1-10, 1995.

ANDERSEN, R.; NEWMAN, J. Societal and individual determinants of medical care utilization in the United States. Health an Society, v. 51, n. 1, p. 95-124, 1973.

ARAÚJO, C. S. et al. Utilização de serviços odontológicos e fatores associados: um estudo de base populacional no Sul do Brasil. Cadernos de Saúde Pública, Rio de Janeiro, v. 25, n. 5, p. 1063-1072, maio, 2009.

ASTROM, A. N. et al. Use of dental services throughout middle and early old ages: a prospective cohort study. Community dentistry and oral epidemiology, Copenhagen, v. 41, p. 30-39, 2013.

BAKER, S. R. Applying Andersen's behavioural model to oral health: what are the contextual factors shaping perceived oral health outcomes? Community dentistry and oral epidemiology, Copenhagen, v. 37, p. 485-494, 2009.

BALDANI, M. H.; ANTUNES, J. L. F. Inequalities in acess and utilization of dental services: a cross-sectional study in an area covered by the Family Health Strategy. Cadernos de Saúde Pública, Rio de Janeiro, v. 27, sup. 2, S272-S283, 2011.

BALDANI, M. H. et al. Determinantes individuais na utilização de serviços odontológicos por adultos e idosos de baixa renda. Revista brasileira de epidemiologia, São Paulo, v. 13, n. 1, p.150-162, 2010.

BARROS, A.J.D.; BERTOLDI, A.D. Desigualdades na utilização e no acesso a serviços odontológicos: uma avaliação em nível nacional. Ciência \& Saúde Coletiva, Rio de Janeiro, v. 7, n. 4, p.709-717, 2002.

BOURDIEU, P. Meditações Pascalianas. 2. ed. Rio de Janeiro: Bertrand Brasil, 2007.

BOURDIEU, P. A distinção: crítica social do julgamento. São Paulo: Edusp; Porto Alegre, RS: Zouk, 2008.

BOURDIEU, P. Razões práticas: sobre a teoria da ação. 10. ed. Campinas, SP: Papirus, 2010.

BRASIL. Ministério da Saúde. Conselho Federal de Odontologia. I Conferência Nacional de Saúde Bucal: relatório final. Brasília, DF, 1986.

BRASIL. Ministério da Saúde. Secretaria de Atenção à Saúde/ Secretaria de Vigilância em Saúde. Departamento de Atenção Básica. Coordenação Geral de Saúde Bucal. SB Brasil 2010: pesquisa nacional de saúde bucal: resultados principais. Brasília, DF, 2011. 
BRASIL. Ministério da Saúde. Departamento de Atenção Básica. Diretrizes da Política Nacional de Saúde Bucal. Brasília, DF, 2004a.

BRASIL. Ministério da Saúde. Secretaria de Atenção à Saúde. Projeto SB Brasil 2003: condições de saúde bucal da população brasileira 2002-2003: resultados principais. Brasília, DF: Ministério da Saúde, 2004b.

CAMARGO, M. B.; DUMITH, S. C.; BARROS, A. J. D. Uso regular de serviços odontológicos entre adultos: padrões de utilização e tipos de serviços. Cadernos de Saúde Pública, Rio de Janeiro, v. 25, n. 9, p. 1894-1906, set. 2009.

CAVALCANTI, R. P.; GASPAR, G. S.; GOES, P. S. A. Utilização e acesso aos serviços de saúde bucal do SUS - uma comparação entre populações rurais e urbanas. Pesquisa brasileira em odontopediatria e clínica integrada, João Pessoa, v. 12, n. 1, p. 121-26, jan./mar. 2012.

CHAVES, S.C.L. et al. Política Nacional de Saúde Bucal: fatores associados à integralidade do cuidado. Rev Saúde Pública, v. 44, n.6, p.1005-1013, 2010.

CHAVES, S.C.L. et al. Avaliação da oferta e utilização de especialidades odontológicas em serviços públicos de atenção secundária na Bahia, Brasil. Cadermos de Saúde Pública, Rio de Janeiro, v. 27, n.1, p.143-154, jan. 2011.

CHAVES, S.C.L. et al. Características do acesso e utilização de serviços odontológicos em municípios de médio porte. Ciência \& Saúde Coletiva, Rio de Janeiro, v. 17, n. 11, p. 3115-3124, 2012.

CHAVES, S.C.L; VIEIRA-DA-SILVA, L.M. Inequalities in oral health pratices and social space: an exploratory qualitativ estudy. Health Policy, v. 86, p. 119-128, 2008.

CRUZ, D.N. Desigualdades na utilização dos serviços odontológicos: posição e tomadas posição no espaço social. 2015. 156f. Tese (Doutorado em Saúde Pública) - Instituto de Saúde Coletiva, Universidade Federal da Bahia, Salvador, 2015.

DAVOGLIO, R.S. et al. Fatores associados a hábitos de saúde bucal e utilização de serviços odontológicos entre adolescentes. Cadernos de Saúde Pública, Rio de Janeiro, v. 25, n. 3, p. $655-667$, mar. 2009.

DINIZ, J. Contribuição ao estudo do sistema incremental de atendimento odontológico. 1987. Tese (Doutorado em Odontologia) - Faculdade de Odontologia, Universidade Federal da Bahia, Salvador, 1987.

DONABEDIAN, A. Los espacios de la salud: aspectos fundamentals de la organización de la atención médica. México, DF: Editora Biblioteca de la Salud: 1988.

FERREIRA, C. O.; ANTUNES, J. L. F.; ANDRADE, F.B. Fatores associados a utilização dos serviços odontológicos por idosos brasileiros. Revista de Saúde Pública, São Paulo, v. 47, supl.3, p. 90-97, dez.2013.

FIGUEIREDO, N.; GOES, P. S. A. Construção da atenção secundária em saúde bucal: um estudo sobre os Centros de Especialidades Odontológicas em Pernambuco, Brasil. Cadernos de Saúde Pública, Rio de Janeiro, v. 25, n. 2, p. 259-267, fev. 2009. 
FRAZÃO, P.; NARVAI, P. C. Saúde Bucal no Sistema Único de Saúde: 20 anos de luta por uma política pública. Saúde em Debate, Rio de Janeiro, v. 33, p. 64-71, 2010.

GOES, P. S. A. et al. Avaliação da atenção secundária nos Centros de Especialidades do Brasil. Cadernos de Saúde Pública, Rio de Janeiro, 28 sup., S81-S89, 2012.

GOMES, A. M. M. et al. Fatores associados ao uso de serviços de saúde bucal: estudo de base populacional em municípios do Maranhão, Brasil. Ciência \& Saúde Coletiva, v. 19, n. 2, p. 629-640, 2014.

GUARNIZO-HERREÑO, C. C. et al. Oral health and welfare estate regimes: a crossnational analysis of European countries. European Journal of Oral Sciences, v. 121, p. 169-175, 2013.

HELLER, A. Teoría de las necesidades en Marx. Barcelona: Ediciones Península, 1986.

IBGE. Acesso e utilização dos serviços de saúde - PNAD 2003. Rio de Janeiro, 2005.

LIMA, A. C. S.; CABRAL, E. D.; VASCONCELOS, M. M. V. B. Satisfação dos usuários assistidos nos Centros de Especialidades Odontológicas no município do Recife, Pernambuco, Brasil. Cadernos de Saúde Pública, Rio de Janeiro, v. 26, n. 5, p. 991-1002, 2010.

MANHÃES, A. L. D.; COSTA, A. J. L. Acesso e utilização de serviços odontológicos no Estado do Rio de Janeiro, Brasil, em 1998: um estudo exploratório a partir da Pesquisa Nacional por Amostra de Domicílios. Cadernos de Saúde Pública, Rio de Janeiro, v. 24, n. 1, p. 207-218, jan. 2008.

MARSHMAN, Z. et al. What influences the use of dental services by adults in the UK? Community Dentistry and Oral Epidemiology, Copenhagen,v. 40, p. 306-314, 2012.

MARTILDES, M. L. R. Avaliação do impacto de programas incrementais de atendimento odontológico sobre as condições de saúde bucal de escolares de 12 anos de idade em São José dos Campos- SP, 1991. 1992. Dissertação (Mestrado em Saúde Pública) - Faculdade de Saúde Pública, Universidade de São Paulo, São Paulo, 1992.

MATSUYAMA, Y. et al. Inequalities of dental prothesis use under universal health care insurance. Community Dentistry and Oral Epidemiology, Copenhagen, v. 42, p. 122-128, 2014.

MIRANDA, C. D. B. C.; PERES, M. A. Determinantes da utilização de serviços odontológicos entre adultos: um estudo de base populacional em Florianópolis, Santa Catarina, Brasil. Cadernos de Saúde Pública, Rio de Janeiro, v. 29, n. 11, p. 2319-2332, nov. 2013.

MUIRHEAD, V. E. et al. Predictors of dental care utilization among working poor Canadians. Community Dentistry and Oral Epidemiology, Copenhagen, v. 37, p. 199-208, 2009.

NARVAI, P. C. Odontologia e saúde bucal coletiva. São Paulo: Hucitec; 1994.

OLIVEIRA, R. S. et al. Use of dental services in áreas covered by the Family Health Strategy in Olinda, Brazil. Cadernos de Saúde Pública, Rio de Janeiro, v. 22, n. 1, p.40-5, 2014. 
OMS. Organização Mundial da Saúde. Diminuindo diferenças: a prática das políticas sobre determinantes sociais da saúde: documento de discussão. Conferência Mundial sobre Determinantes Sociais da Saúde. Rio de Janeiro, 2011.

PAIM, J. S. Políticas de Saúde no Brasil. In: ROUQUAYROL, M.Z; ALMEIDA FILHO, N. Epidemiologia \& Saúde, 6. ed. Rio de Janeiro: MEDSI, 2003. p. 587-603.

PALÈNCIA, L. et al. Socioeconomic inequalities in the use of dental care services in Europe: what is the role of public coverage? Community Dentistry and Oral Epidemiology, Copenhagen, v. 42, p. 97-105, 2014.

PAVÃO, A. L. B.; COELI, C. M. Modelos teóricos do uso de serviços de saúde: conceitos e revisão. Cadernos de Saúde Coletiva, Rio de Janeiro, v. 16, n. 3, p. 471-482, 2008.

PEREIRA, C. O. et al. Impacto da Estratégia Saúde da Família sobre indicadores de saúde bucal: análise em municípios do Nordeste brasileiro com mais de 100 mil habitantes. Cadernos de Saúde Pública, Rio de Janeiro, v. 28, n. 3, p. 449-462, mar. 2012.

PEREIRA, C. R. S. et al. Impacto da Estratégia Saúde da Família com equipe de saúde bucal sobre a utilização de serviços odontológicos. Cadernos de Saúde Pública, Rio de Janeiro, v. 25 , n. 5, p.985-996, maio 2009.

PERES, K. G. et al. Redução das desigualdades sociais na utilização de serviços odontológicos no Brasil entre 1998 e 2008. Revista Saúde Pública, Rio de Janeiro, v. 46, n. 2, p.250-8, 2012a.

PERES, M. A. et al. Desigualdades no acesso e na utilização de serviços odontológicos no Brasil: análise do Sistema de vigilância de fatores de risco e proteção para doenças crônicas por inquérito telefônico (VIGITEL 2009). Cadernos de Saúde Pública, Rio de Janeiro, 28 Sup., S9o-S100, 2012b.

PINTO, R. S.; MATOS, D. L.; LOYOLA FILHO, A. I. Características associadas ao uso de serviços odontológicos públicos pela população adulta brasileira. Ciência \& Saúde Coletiva, Rio de Janeiro, v. 17, n. 2, p. 531-544, 2012.

ROCHA, R. A. C. P.; GOES, P. S. A. Comparação de acesso aos serviços de saúde bucal em áreas cobertas e não cobertas pela Estratégia Saúde da Família em Campina Grande, Paraíba, Brasil. Cadernos de Saúde Pública, Rio de Janeiro, v. 24, n. 12, p. 2871-2880, dez. 2008.

RODRIGUES, L. A. M. et al. Uso de serviços odontológicos entre pré-escolares: estudo de base populacional. Ciência \& Saúde Coletiva, Rio de Janeiro, v. 19, n. 10, p. 4247-4256, 2014. SCHRAIBER, L. B.; MENDES-GONÇALVES, R. B. Necessidades de saúde e atenção primária. In: SCHRAIBER, L. B.; NEMES, M. I. B.; MENDES-GONÇALVES, R. B. Saúde do adulto: programas e ações na unidade básica. São Paulo: Hucitec, 1996. cap 1.

SILVA, Z. P. et al. Perfil sociodemográfico e padrão de utilização dos serviços de saúde do Sistema Único de Saúde (SUS), 2003-2008. Ciência \& Saúde Coletiva, Rio de Janeiro, n. 16, v.9, p.3807-3816, 2011. 
SOARES, F. F.; CHAVES, S. C. L.; CANGUSSU, M. C. T. Governo local e serviços odontológicos: análise da desigualdade na utilização. Cadernos de Saúde Pública, Rio de Janeiro, v. 31, n. 3, p. 586-596, 2015.

SOARES, F. F.; CHAVES, S. C. L.; CANGUSSU, M. C. T. Desigualdades na utilização de serviços de saúde bucal na atenção básica e fatores associados em dois municípios brasileiros. Revista panamericana de salud pública, Washington, D.C., v. 6 , n. 34, p. 401-406, 2013.

SOUZA, L. F.; CHAVES, S. C. L. Política Nacional de Saúde Bucal: acessibilidade e utilização de serviços odontológicos especializados em um município de médio porte na Bahia. Revista Baiana de Saúde Pública, Salvador, n. 34, v. 2, p. 371-378, abr./jun. 2010.

TCHICAYA, A.; LORENTZ, N. Socioeconomic inequalities in the non-use of dental care in Europe. International Journal for Equity in Health, n. 13, v. 7, p. 1-14, 2014.

TRAVASSOS, C.; MARTINS, M. Uma revisão sobre os conceitos de acesso e utilização de serviços de saúde. Cadernos de Saúde Pública, Rio de Janeiro, n. 20, supl. 2, S19o-S198, 2004.

VIEIRA-DA-SILVA, L. M. Saúde e Espaço Social. In: NOGUEIRA, R. P. (Org.). Determinação social da saúde e reforma sanitária. Rio de Janeiro: CEBES. 2010. p.180-200.(Coleção Pensar em Saúde). 


\begin{tabular}{|c|c|c|c|c|}
\hline Autor, ano & Local & População & Aspectos metodológicos & Resultados principais \\
\hline $\begin{array}{l}\text { Araújo et al., } \\
2009\end{array}$ & Pelotas-RS & $\begin{array}{l}\mathrm{n}=4.226 \text { indivíduos de } 10 \\
\text { anos ou mais. }\end{array}$ & $\begin{array}{l}\text { Estudo transversal de base } \\
\text { populacional com aplicação } \\
\text { de questionários. }\end{array}$ & $\begin{array}{l}\text { 50,9\% utilizaram serviço no último ano. Ter } \\
\text { escolaridade, sermaior de oito anos, maior } \\
\text { nível socioeconômico e maior percepção de } \\
\text { problemas aumentaram o uso. }\end{array}$ \\
\hline $\begin{array}{l}\text { Camargo; } \\
\text { Dumith; Barros, } \\
2009\end{array}$ & Pelotas-RS & $\mathrm{n}=2.961$ adultos. & $\begin{array}{l}\text { Estudo transversal com } \\
\text { aplicação de questionário. }\end{array}$ & $\begin{array}{l}\text { 32,8\% faziam uso regular. Esse uso foi maior } \\
\text { no sexo feminino, menor de } 60 \text { anos, de maior } \\
\text { escolaridade e nível socioeconômico e usuário de } \\
\text { serviços privados. }\end{array}$ \\
\hline $\begin{array}{l}\text { Davoglio et al., } \\
2009\end{array}$ & Gravataí-RS & $\mathrm{n}=1.170$ adolescentes. & $\begin{array}{c}\text { Estudo transversal com } \\
\text { aplicação de questionário } \\
\text { sobre hábitos de saúde bucal } \\
\text { e estilo de vida. }\end{array}$ & $\begin{array}{l}68,9 \% \text { utilizaram o serviço no último ano. Esse } \\
\text { foi maior no nível socioeconômico mais alto. }\end{array}$ \\
\hline $\begin{array}{l}\text { Figueiredo \& } \\
\text { Góes, } 2009\end{array}$ & $\begin{array}{l}\text { Estado de } \\
\text { Pernambuco }\end{array}$ & $\mathrm{n}=22$ CEO. & $\begin{array}{c}\text { Série histórica dos } \\
\text { procedimentos do Sistema } \\
\text { de Informação Ambulatorial } \\
\text { (SIA) do SUS, } 2006 \\
\text { (utilização de procedimentos } \\
\text { especializados). }\end{array}$ & $\begin{array}{l}\text { 40,9\% dos CEO com bom desempenho de } \\
\text { metas. Quanto menor o porte populacional } \\
\text { pior o desempenho. Quanto maior o Índice } \\
\text { de Desenvolvimento Humano, melhor o } \\
\text { desempenho. }\end{array}$ \\
\hline $\begin{array}{l}\text { Pereira et al., } \\
2009\end{array}$ & Natal-RN & $\begin{array}{l}\mathrm{n}=4.604 \text { indivíduos de } \\
\text { todas as idades. }\end{array}$ & $\begin{array}{l}\text { Comparação da percepção de } \\
\text { necessidade de tratamento } \\
\text { odontológico entre áreas } \\
\text { cobertas pela ESB, UBS } \\
\text { tradicional e sem serviços de } \\
\text { saúde bucal. }\end{array}$ & $\begin{array}{l}\text { Áreas cobertas pela ESB tem maior utilização } \\
\text { quando comparadas às áreas sem cobertura. } \\
\text { Utilização de } 1,32 \% \text { na ausência de serviço de } \\
\text { saúde; } 17,5 \% \text { nas UBS; e } 45,4 \% \text { na ESF. }\end{array}$ \\
\hline
\end{tabular}


Quadro 1 - Estudos nacionais publicados entre 2009-2015 sobre utilização dos serviços odontológicos no Brasil

\begin{tabular}{|c|c|c|c|c|}
\hline $\begin{array}{l}\text { Baldani et al., } \\
2010\end{array}$ & $\begin{array}{c}\text { Ponta Grossa- } \\
\text { PR }\end{array}$ & $\begin{array}{c}\mathrm{n}=246 \text { indivíduos de } 35 \\
\text { anos ou mais. }\end{array}$ & $\begin{array}{c}\text { Estudo transversal com } \\
\text { aplicação de questionário. }\end{array}$ & $\begin{array}{l}38,1 \% \text { adultos e } 19,5 \% \text { dos idosos foram no } \\
\text { dentista no último ano. Usar prótese total, não } \\
\text { residir em domicílio próprio e ter pior higiene } \\
\text { oral foram associadas como o maior uso. }\end{array}$ \\
\hline $\begin{array}{l}\text { Souza \& } \\
\text { Chaves, } 2010\end{array}$ & $\begin{array}{l}\text { Sem } \\
\text { identificação- } \\
\text { Bahia }\end{array}$ & $\mathrm{n}=148$ de 3 a 75 anos. & $\begin{array}{l}\text { Estudo transversal com } \\
\text { aplicação de questionário para } \\
\text { identificar acesso ao CEO. }\end{array}$ & $\begin{array}{l}\text { 61\% utilizou a atenção básica. Maior acesso } \\
\text { ao CEO por usuários que vinham da atenção } \\
\text { básica e para periodontia em relação a outras } \\
\text { especialidades. }\end{array}$ \\
\hline $\begin{array}{l}\text { Chaves et al., } \\
2011\end{array}$ & $\begin{array}{l}\text { Sem } \\
\text { identificação- } \\
\text { Bahia }\end{array}$ & $\mathrm{n}=4$ CEO. & $\begin{array}{l}\text { Produção ambulatorial, oferta } \\
\text { e utilização dos serviços } \\
\text { através do SIA/SUS, ano } 2008 \text {. }\end{array}$ & $\begin{array}{l}\text { Utilização de endodontias variaram de } 13 \% \text { a } \\
\text { 33,4\% e a maior utilização foi da periodontia } \\
\text {-100\%. Menor utilização quando existem } \\
\text { barreiras de acesso. }\end{array}$ \\
\hline Silva et al., 2011 & Brasil & $\begin{array}{l}\mathrm{n}=384.834 \text { indivíduos em } \\
2003 . \\
\mathrm{n}=391.868 \text { indivíduos em } \\
2008 .\end{array}$ & $\begin{array}{l}\text { Dados da PNAD } 2003 \text { e } 2008 \\
\text { sobre utilização de serviços de } \\
\text { saúde bucal. }\end{array}$ & $\begin{array}{c}\text { Utilização dos serviços odontológicos de } \\
\text { 8,6\% em } 2003 \text { e 14,3\% em } 2008 \text {. As consultas } \\
\text { aconteceram de forma predominante nos } \\
\text { serviços não SUS, com menor proporção na } \\
\text { região Nordeste. }\end{array}$ \\
\hline $\begin{array}{l}\text { Cavalcanti; } \\
\text { Gaspar; Góes, } \\
2012\end{array}$ & $\begin{array}{l}\text { Sem } \\
\text { idenfiticação- } \\
\text { Bahia }\end{array}$ & $\begin{array}{c}\text { n=949 município A e } \\
\text { n=1.049 município B } \\
\text { Idade/Faixa índice OMS }\end{array}$ & $\begin{array}{c}\text { Estudo transversal com } \\
\text { aplicação de questionário. }\end{array}$ & $\begin{array}{c}\text { Utilização de } 80 \% \text { no município A e } 67,6 \% \text { no } \\
\text { município B. Maior utilização na zona urbana e } \\
\text { em idades maiores. }\end{array}$ \\
\hline $\begin{array}{l}\text { Chaves et al., } \\
2012\end{array}$ & $\begin{array}{l}\text { Não } \\
\text { identificado- } \\
\text { Bahia }\end{array}$ & $\begin{array}{c}\mathrm{n}=2.539 \text { indivíduos de } 15 \\
\text { anos oumais. }\end{array}$ & $\begin{array}{c}\text { Estudo transversal com } \\
\text { aplicação de questionário. }\end{array}$ & $\begin{array}{c}\text { Utilização na atenção básica foi } 26 \% \text { e } \\
\text { especializada } 11,7 \% \text {. Pouca articulação entre os } \\
\text { dois níveis de atenção. }\end{array}$ \\
\hline
\end{tabular}




\begin{tabular}{|c|c|c|c|c|}
\hline Góes et al., 2012 & Brasil & $n=613$ CEO. & $\begin{array}{l}\text { Série histórica dos } \\
\text { procedimentos SIA/SUS, } 2007 \\
\text { (utilização de procedimentos } \\
\text { especializados) - } \\
\text { cumprimento das metas. }\end{array}$ & $\begin{array}{c}\text { Melhor cumprimento das metas (utilização) em } \\
\text { cidades com maior porte populacional e maior } \\
\text { cobertura da ESF. }\end{array}$ \\
\hline $\begin{array}{l}\text { Pereira et al., } \\
2012\end{array}$ & $\begin{array}{l}12 \text { municípios } \\
\text { com mais } \\
\text { de } 100 \text { mil } \\
\text { habitantes - } \\
\text { Nordeste }\end{array}$ & $\mathrm{n}=59.221$ indivíduos. & $\begin{array}{c}\text { Utilização dos serviços entre } \\
\text { áreas cobertas pela ESB, UBS } \\
\text { tradicional e sem serviços de } \\
\text { saúde bucal. }\end{array}$ & $\begin{array}{l}\text { Maior acesso e utilização de serviços preventivos } \\
\text { em áreas cobertas pela ESB. Ações curativas } \\
\text { foram mais utilizadas no modelo tradicional. }\end{array}$ \\
\hline $\begin{array}{l}\text { Peres et al., } \\
2012 \mathrm{a}\end{array}$ & Brasil & $\begin{array}{l}\mathrm{n}=384.834 \text { indivíduos em } \\
2003 . \\
\mathrm{n}=391.868 \text { indivíduos em } \\
2008 .\end{array}$ & $\begin{array}{c}\text { Dados da PNAD } 2003 \text { e } 2008 \\
\text { sobre utilização de serviços de } \\
\text { saúde bucal. Amostra dividida } \\
\text { em quintis. }\end{array}$ & $\begin{array}{l}\text { Aumento do uso de serviços entre } 2003 \text { e } 2008 . \\
\text { Menor utilização em idade inferior a seis anos e } \\
\text { entre os mais pobres. }\end{array}$ \\
\hline $\begin{array}{l}\text { Peres et al., } \\
2012 b\end{array}$ & $\begin{array}{l}\text { Capitais } \\
\text { brasileiras }\end{array}$ & $\mathrm{n}=54.367$ & Inquérito telefônico. & $\begin{array}{l}\text { Utilização do SUS de } 6,2 \% \text { (no DF) a } 35,2 \% \text { (Boa } \\
\text { Vista). Serviços privados foram utilizados por } \\
61,1 \% \text { da amostra. Maior uso em mulheres, meia } \\
\text { idade e mais escolarizados. }\end{array}$ \\
\hline $\begin{array}{l}\text { Pinto; Matos; } \\
\text { Loyola Filho, } \\
2012\end{array}$ & Brasil & $\begin{array}{c}\mathrm{n}=12.425 \text { adultos de } 35- \\
44 \text { anos. }\end{array}$ & Dados do SB Brasil '²003. & $\begin{array}{l}\text { 51,8\% fizeram uso de serviços públicos. O uso } \\
\text { do serviço público foi associado à menor renda, } \\
\text { municípios de menor porte, moradores de zona } \\
\text { rural e menor escolaridade quando comparado } \\
\text { ao serviço privado. }\end{array}$ \\
\hline
\end{tabular}


Quadro 1 - Estudos nacionais publicados entre 2009-2015 sobre utilização dos serviços odontológicos no Brasil

(conclusão)

\begin{tabular}{|c|c|c|c|c|}
\hline $\begin{array}{l}\text { Ferreira; } \\
\text { Antunes; } \\
\text { Andrade, } 2013\end{array}$ & Brasil & $\begin{array}{c}\mathrm{n}=6.702 \text { idosos de } 65-74 \\
\text { anos. }\end{array}$ & Dados do SB Brasil 2010. & $\begin{array}{l}\text { 46,5\% fizeram uso nos últimos dois anos. Maior } \\
\text { uso entre aqueles com maior escolaridade e } \\
\text { renda e não usuários de prótese. }\end{array}$ \\
\hline $\begin{array}{l}\text { Soares; Chaves; } \\
\text { Cangussu, } 2013\end{array}$ & $\begin{array}{l}\text { Sem } \\
\text { identificação } \\
\text {-Bahia }\end{array}$ & $\begin{array}{c}\mathrm{n}=2.539 \text { indivíduos acima } \\
\text { de } 15 \text { anos. }\end{array}$ & $\begin{array}{l}\text { Estudo transversal com } \\
\text { aplicação de questionário. }\end{array}$ & $\begin{array}{l}26,86 \% \text { utilizaram o serviço SUS no último } \\
\text { ano. A exodontia foi mais utilizada em usuário } \\
\text { beneficiado por programa social, acima de } 35 \\
\text { anos e de menor escolaridade. }\end{array}$ \\
\hline $\begin{array}{l}\text { Miranda \& } \\
\text { Peres, } 2013\end{array}$ & $\begin{array}{l}\text { Florianópoli- } \\
\text { SC }\end{array}$ & $\mathrm{n}=1.729$ adultos & $\begin{array}{l}\text { Estudo transversal com } \\
\text { aplicação de questionário. }\end{array}$ & $\begin{array}{c}\text { 66\% fizeram uso de serviços. Uso maior entre as } \\
\text { mulheres, nos mais escolarizados e portadores } \\
\text { de planos privados. }\end{array}$ \\
\hline $\begin{array}{l}\text { Gomes et al., } \\
2014\end{array}$ & $\begin{array}{l}\text { Municípios } \\
\text { com mais } \\
\text { de } 100 \text { mil } \\
\text { habitantes - } \\
\text { Maranhão }\end{array}$ & $\begin{array}{l}\mathrm{n}=1.214 \text { crianças; } \mathrm{n}= \\
1.059 \text { adultos. }\end{array}$ & $\begin{array}{l}\text { Estudo transversal com } \\
\text { aplicação de questionário. }\end{array}$ & $\begin{array}{l}\text { Uso por } 9 \% \text { crianças e } 28,1 \% \text { adultos. Uso foi } \\
\text { associado com maior idade, maior escolaridade e } \\
\text { o fato de referir necessidade de tratamento. }\end{array}$ \\
\hline $\begin{array}{l}\text { Rodrigues et al., } \\
2014\end{array}$ & $\begin{array}{l}\text { Montes } \\
\text { Claros-MG }\end{array}$ & $\begin{array}{c}\mathrm{n}=809 \text { crianças de 18-36 } \\
\text { meses. }\end{array}$ & $\begin{array}{l}\text { Estudo transversal com } \\
\text { aplicação de questionário. }\end{array}$ & $\begin{array}{l}\text { 15,5\% utilizaram serviço no último ano. O uso foi } \\
\text { maior em crianças mais velhas, de maior renda, } \\
\text { em famílias com moradia própria e com maior } \\
\text { acesso à informação em saúde. }\end{array}$ \\
\hline $\begin{array}{l}\text { Soares; Chaves; } \\
\text { Cangussu, } 2015\end{array}$ & $\begin{array}{l}\text { Sem } \\
\text { identificação- } \\
\text { Bahia }\end{array}$ & $\begin{array}{c}\mathrm{n}=2.539 \text { indivíduos acima } \\
\text { de } 15 \text { anos. }\end{array}$ & $\begin{array}{l}\text { Estudo transversal com } \\
\text { aplicação de questionário. }\end{array}$ & $\begin{array}{c}\text { No último ano, } 38,76 \% \text { utilizaram o serviço } \\
\text { privado, } 33,80 \% \text { a atenção básica no SUS e } \\
17,29 \% \text { a atenção básica e a especializada. } \\
\text { A menor escolaridade implicou maior uso do } \\
\text { serviço público. }\end{array}$ \\
\hline
\end{tabular}

${ }^{1}$ Inquéritos epidemiológicos em saúde bucal, realizados nacionalmente em 2003 e 2010 conhecidos por SB Brasil. 